\title{
Evaluation of the potential of chicoric acid as a natural food antioxidant
}

\author{
XUELIANG ZHU ${ }^{1,2}$, FENGHONG HUANG $^{2}$, XIA XIANG $^{2}$, MIN FAN $^{3}$ and TINGTAO CHEN ${ }^{4}$ \\ ${ }^{1}$ State Key Laboratory of Advanced Technology for Materials Synthesis and Processing, Wuhan University of Technology, \\ Wuhan, Hubei 430070; ${ }^{2}$ Oil Crops Research Institute of The Chinese Academy of Agricultural Sciences, \\ Hubei Key Laboratory of Lipid Chemistry and Nutrition, Wuhan, Hubei 430062; ${ }^{3}$ Institute of Clinical Nutrition, \\ The Xinjiang Uygur Autonomous Region People's Hospital, Urumqi, Xinjiang Uygur 830000; \\ ${ }^{4}$ Institute of Translational Medicine, Nanchang University, Nanchang, Jiangxi 330031, P.R. China
}

Received January 8, 2018; Accepted July 13, 2018

DOI: $10.3892 /$ etm.2018.6596

\begin{abstract}
Dietary fat is important for human health, while its excessive consumption or the oxidation of lipids may cause various diseases. In the present study, the potential of chicoric acid (CA) as a natural food antioxidant was evaluated. To evaluate this, spectrophotometry analysis measured CA antioxidant activity, the analytical method of the sanitary standard of edible lard and rapeseed oil measured CA anti-oil oxidation, the Oxford cup method measured CA anti-microbial activity, Oil red $\mathrm{O}$ staining assessed intracellular triglycerides following CA administration, and RT-qPCR analysis and ELISA assessed CA anti-inflammatory activity. The results indicated that CA had a marked scavenging capacity for the 2,2-diphenyl-1-picrylhydrazyl radical, as well as a reductive action, and after incubation for 5 days, $0.05 \% \mathrm{CA}$ achieved a significantly higher peroxide value than $0.02 \%$ tert-butylhydroquinone used as a reference $(\mathrm{P}<0.05)$. In addition, $160 \mathrm{nM}$ CA inhibited the growth of a variety of common pathogenic microbes in humans, significantly inhibited fat droplet formation $(\mathrm{P}<0.05)$ and reduced the production of interleukin- $1 \beta$ and tumor necrosis factor- $\alpha$ in a dose-dependent manner $(\mathrm{P}<0.05)$. Therefore, functional foods containing CA may be used as natural antioxidant supplements to prevent the oxidation of oil and protect human health.
\end{abstract}

Correspondence to: Dr Tingtao Chen, Institute of Translational Medicine, Nanchang University, 1299 Xuefu Road, Honggu, Nanchang, Jiangxi 330031, P.R. China

E-mail: chentingtao1984@163.com

Professor Min Fan, Institute of Clinical Nutrition, The Xinjiang Uygur Autonomous Region People's Hospital, 91 Tianchi Road, Tianshan, Urumqi, Xinjiang Uygur 830000, P.R. China

E-mail: xjfanmin2000@163.com

Key words: chicoric acid, natural food antioxidant, lipids, anti-bacterial activity

\section{Introduction}

Fat is an important component of a healthy human diet and is essential for the synthesis of hormones, as well as the delivery of essential vitamins throughout the body (1). However, excessive consumption of dietary fat may increase the cholesterol levels in the human body, enhancing the chance of heart disease, cancer and type 2 diabetes (2).

It is well known that lipids may be oxidised in vitro or in vivo by multiple oxidants, the products of which are key factors in conditions including atherosclerosis, neurological disorders, cancer and aging (3-5). Although oxidation may trigger a chain of chemical reactions and damage cells, certain antioxidants are able to terminate these chain reactions, exerting beneficial effects with regard to health maintenance and disease prevention (6-8). Oxidation due to exposure to oxygen and sunlight makes unsaturated fats turn rancid and become discoloured, and therefore, antioxidants are widely used as food additives to protect against degradation (9).

It has been reported that certain plants (e.g., berries) contain abundant and numerous bioactive compounds, natural phytochemicals, which may be used as antioxidants to protect lipids from undesired oxidative modification $(3,6)$. Chicoric acid (CA) is a caffeic acid derivative, which extensively occurs in certain edible plants and vegetables, including chicory (Cichorium intybus), lettuce and those of the genera Echinacea and dandelion (Taraxacum). CA has been regarded as a nutraceutical with potent antioxidant activities $(10,11)$, and previous studies have indicated that CA attenuated inflammation through suppression of the phosphoinositide-3 kinase/Akt and nuclear factor- $\kappa \mathrm{B}$ signalling pathways to markedly inhibit a high-fat diet-induced inflammatory response $(12,13)$. However, the potential of CA as a natural food antioxidant has remained to be determined.

In the present study, the antioxidant effects and inhibition of foodborne pathogens by CA was systematically evaluated in vitro, and the attenuation of inflammation as well as the inhibition of lipid droplet formation (which evaluated the inhibition of adipogenic differentiation in pre-adipogenic cells and the regulation of lipid metabolism by adipocytes) was studied 
using RAW264.7 and 3T3-L1 cells, respectively. To the best of our knowledge, the present study was the first to investigate the potential use of CA as a natural food antioxidant.

\section{Materials and methods}

Antioxidant activity of $C A$. The clearance effect of CA on the 2,2-diphenyl-1-picrylhydrazyl (DPPH) radical was estimated as follows: Vincristine (VC) or CA at 95,190 or $380 \mathrm{mg} / \mathrm{ml}$ was mixed with $100 \mathrm{mM}$ Tris- $\mathrm{HCl}$ buffer $(\mathrm{pH}=7.5)$ and then added to $1 \mathrm{ml}$ of $500 \mu \mathrm{M}$ DPPH in ethanol $(250 \mu \mathrm{M})$. The mixture was vigorously agitated at room temperature in the dark and the absorbance of the resulting solution was spectrophotometrically determined by measuring the absorption at $517 \mathrm{~nm}(14,15)$.

To determine the $\cdot \mathrm{OH}$ radical scavenging activity, $1 \mathrm{ml}$ of reaction mixture containing $2.8 \mathrm{mM}$ deoxyribose, $20 \mathrm{mM}$ phosphate buffer (pH 7.4), CA or VC $(95,190$ or $380 \mathrm{mg} / \mathrm{ml})$, $100 \mathrm{mM} \mathrm{FeCl}_{3}, 104 \mathrm{mM}$ EDTA, $1 \mathrm{mM} \mathrm{H}_{2} \mathrm{O}_{2}$ and $100 \mathrm{mM}$ ascorbic acid was incubated at $37^{\circ} \mathrm{C}$ for $60 \mathrm{~min}$. Subsequently, $50 \mathrm{ml} \mathrm{2 \%}(\mathrm{w} / \mathrm{v})$ butylated hydroxytoluene, $1 \mathrm{ml} \mathrm{2.8 \% (w/v)}$ trichloroacetic acid and $1 \mathrm{ml} 1 \%(\mathrm{w} / \mathrm{v}) 2$-thiobarbituric acid were added and the mixture was incubated at $80^{\circ} \mathrm{C}$ for $20 \mathrm{~min}$. The reaction was terminated by incubation in an ice-water bath for $5 \mathrm{~min}, 2 \mathrm{ml} \mathrm{n}$-butanol was added to each tube and the degree of deoxyribose degradation was spectrophotometrically determined by measuring the absorption at $532 \mathrm{~nm}(16)$.

To assess the $\mathrm{Fe}^{2+}$ chelation ability, CA or VC solution was mixed with $2 \mathrm{mM} \mathrm{FeCl}_{2}$ and $5 \mathrm{mM}$ ferrozine at a ratio of 10:1:2 in PBS. After incubation for $10 \mathrm{~min}$ at room temperature, the absorbance was measured at $562 \mathrm{~nm}$ (14).

The oxygen reduction ability of CA was determined as follows: $\mathrm{CA}$ or $\mathrm{VC}$ solution was mixed with potassium ferricyanide (the final concentration was $0.5 \%$ ) in phosphate buffer (pH 6.6). The mixture was incubated at $50^{\circ} \mathrm{C}$ for $20 \mathrm{~min}$. Subsequently, an equal volume of $1 \%$ trichloroacetic acid was added to the mixture, which was centrifuged at $5,000 \mathrm{x} \mathrm{g}$ for $10 \mathrm{~min}$. The upper phase was mixed with distilled water and $0.1 \% \mathrm{FeCl}_{3}$ at a ratio of 1:1:2, and the absorbance was measured at $700 \mathrm{~nm}$ (14).

Anti-oil oxidation effect of CA. CA was added to $30 \mathrm{~g}$ rapeseed oil (Shandong Luhua Group Co., Ltd., Laiyang, China) to reach the final concentrations of 0.02 and $0.05 \%$. Pure rapeseed oil was used as a control group and rapeseed oil with $0.02 \%$ tert-butylhydroquinone (TBHQ) was used as a positive control. All samples were heated to $60^{\circ} \mathrm{C}$ over $30 \mathrm{~min}$ and then maintained at this temperature. The peroxide value (POV) of each system was measured after 1, 3 and 5 days. The POV was tested using the analytical method of the sanitary standard of edible lard and rapeseed oil, People's Republic of China (GB/T5009.37-1996) (17).

Anti-microbial activity of $C A$. The agar diffusion assay was performed to examine the anti-microbial activity of different concentrations of CA on Lactobacillus plantarum (isolated from human feces; State Key Laboratory of Food, Nanchang University, Nanchang, China), L. rhamnosus (Culturelle; i-Health, Inc., Cromwell, CT, USA), L. crispatus (isolated from vaginal secretion of women; Institute of Translational Medicine, Nanchang University) and Bifidobacterium longum isolated from a probiotic drug (Bifico; Shanghai Xinyi Pharmaceutical Factory Co., Ltd., Shanghai, China), as well as Salmonella enteritidis, Shigella typhimurium, Escherichia coli and Listeria monocytogenes (isolated from human feces; State Key Laboratory of Food, Nanchang University). The isolation of the microorganisms was performed as reported by previous studies by our group (15,18-22). Cultures of the probiotics L. plantarum, L. rhamnosus, L. crispatus, B. longum grown anaerobically for $24 \mathrm{~h}$ in De Man, Rogosa and Sharpe medium (Oxoid Ltd; Thermo Fisher Scientific, Inc., Waltham, MA, USA) were centrifuged at $8,000 \times \mathrm{g}$ for $10 \mathrm{~min}$ at $4^{\circ} \mathrm{C}$ to obtain supernatants. Overnight $(12 \mathrm{~h})$ cultures of pathogenic microorganisms including $S$. enteritidis, S. typhimurium, $E$. coli and L. monocytogenes were spread on the surface of the Lysogeny Broth agar (Oxoid, Ltd.; Thermo Fisher Scientific, Inc.) plates. Aliquots $(200 \mu \mathrm{l})$ of the supernatant were loaded into an Oxford cup (outer diameter, $7.8 \pm 0.1 \mathrm{~mm}$; inner diameter, $6.0 \pm 0.1 \mathrm{~mm}$; height, $10.0 \pm 0.1 \mathrm{~mm}$ ), which was placed on the surface of the agar. The size of the clear zone formed around the Oxford cup was measured.

Oil red $O$ staining for intracellular triglycerides. 3T3-L1 cells (Pre-adipocytes derived from mouse 3T3 cells that is used in biological research on adipose tissue; ATCC-CL-173; American Type Culture Collection, Manassas, VA, USA) were maintained in Dulbecco's modified Eagle's medium (DMEM) supplemented with 10\% fetal bovine serum (FBS; both Gibco; Thermo Fisher Scientific, Inc.) and antibiotics $\left(100 \mathrm{U} / \mathrm{ml}\right.$ penicillin and $100 \mu \mathrm{g} / \mathrm{ml}$ streptomycin) at $37^{\circ} \mathrm{C}$ in a humidified atmosphere containing $5 \% \mathrm{CO}_{2}$, and intracellular lipid accumulation in adipocytes was assessed using Oil Red O staining. In brief, In brief, 3T3-L1 cells were cultured in a 6-well plate at a density of $1 \times 10^{5}$ cells/well and CA was incubated with 3T3-L1 cells at final concentrations of $12.5,25$ or $50 \mu \mathrm{M}$ for $24 \mathrm{~h}$ at $37^{\circ} \mathrm{C}$ in a $5 \% \mathrm{CO}_{2}$ incubator. The cells were gently washed with ice-cold PBS (pH 7.4) twice and fixed with $10 \%$ formalin at room temperature for at least $30 \mathrm{~min}$. Subsequently, the wells were washed with $60 \%$ isopropyl alcohol for $5 \mathrm{~min}$ and then exhaustively with PBS. The wells were allowed to dry completely prior to the addition of filtered Oil Red O solution, followed by incubation for $30 \mathrm{~min}$ at room temperature. The stained lipid droplets in 3T3-L1 pre-adipocytes were exhaustively rinsed three times with PBS. Stained oil droplets were extracted with $100 \%$ isopropanol for $10 \mathrm{~min}$ to quantify intracellular lipids. The dye extract was then immediately removed by gentle pipetting and its absorbance was measured spectrophotometrically at $520 \mathrm{~nm}$. The amount of Oil Red O-stained material (OROSM, \%) was compared to that in control wells containing cell culture medium without $\mathrm{CA}$ and calculated according to the following formula: OROSMe $\%=$ absorbance of tested sample extract/absorbance without tested sample extract x100 (23).

Anti-inflammatory effects of CA. As primary macrophages isolated from humans are difficult to culture due to their high sensitivity to culture conditions, which makes them 

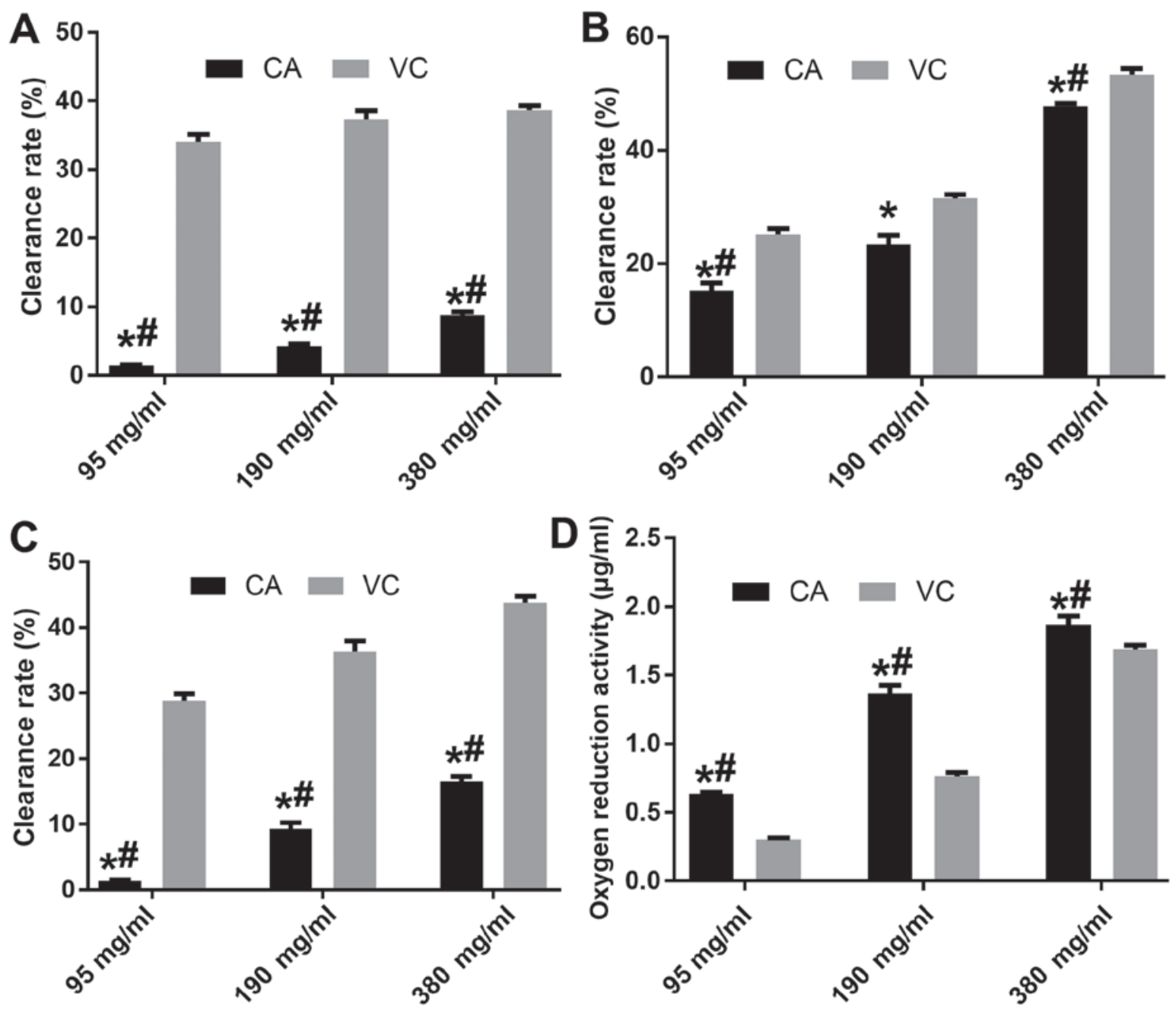

Figure 1. Anti-oxidative activity of different concentrations of CA. Clearance of (A) $\cdot \mathrm{OH}$; (B) DPPH; (C) $\mathrm{Fe}^{2+}$ chelation. (D) Oxygen-reduction activity. Values are expressed as the mean \pm standard deviation. ${ }^{\prime} \mathrm{P}<0.05 \mathrm{vs} .95 \mathrm{mg} / \mathrm{ml} \mathrm{VC}$; ${ }^{~} \mathrm{P}<0.05$ vs. the same concentration of VC. CA, chicoric acid; DPPH, 2,2-diphenyl-1-picrylhydrazyl radical; VC, vincristine.

prone to losing their biological characteristics (24), the RAW264.7 murine macrophage cell line (passage 8-20; ATCCTIB-71; American Type Culture Collection) was used in the present study. RAW264.7 cells were maintained in DMEM supplemented with $10 \% \mathrm{FBS}$ and antibiotics (100 U/ml penicillin and $100 \mu \mathrm{g} / \mathrm{ml}$ streptomycin) at $37^{\circ} \mathrm{C}$ in a $5 \% \mathrm{CO}_{2}$ incubator.

RAW 264.7 cells were seeded into 24-well plates ( $5 \times 10^{4}$ cells per well), allowed to attach for $24 \mathrm{~h}$ and then washed with PBS. Cells were treated with PBS (Control group), lipopolysaccharide (LPS; $1 \mu \mathrm{g} / \mathrm{ml}$ each), LPS $+5 \mu \mathrm{M}$ $\mathrm{CA}, \mathrm{LPS}+10 \mu \mathrm{M}$ CA, LPS $+20 \mu \mathrm{M}$ CA or LPS $+40 \mu \mathrm{M} \mathrm{CA}$ for $24 \mathrm{~h}$, and the supernatants were harvested. The amounts of interleukin (IL)-1 $\beta$ and tumor necrosis factor (TNF)- $\alpha$ secreted into the cell supernatants were determined using ELISA kits for IL-1 $\beta$ (cat. no. 88-7013) and TNF- $\alpha$ (cat. no. 88-7324; both Thermo Fisher Scientific, Inc., Waltham, MA, USA).

Statistical analysis. Values are expressed as mean \pm standard deviation. Data were analyzed with SPSS software (version 30.0; IBM Corp., Armonk, NY, USA) using one-way analysis of variance followed by the least-significant difference post-hoc test. $\mathrm{P}<0.05$ was considered to indicate a statistically significant difference.

\section{Results}

Anti-oxidative activity of $C A$. The anti-oxidative activity of different doses of CA was evaluated with VC used at the same doses as a positive control (Fig. 1). The results indicated that CA had a dose-dependent anti-oxidative activity, and that the clearance effect of $\mathrm{CA}$ on $\cdot \mathrm{OH}, \mathrm{DPPH}$ and $\mathrm{Fe}^{2+}$ was weaker than that of VA at the same concentration $(\mathrm{P}<0.05)$, while $380 \mathrm{mg} / \mathrm{ml} \mathrm{CA}$ possessed a better clearance effect of DPPH compared with $95 \mathrm{mg} / \mathrm{ml} \mathrm{VC} \mathrm{(Fig.} \mathrm{1A-C).} \mathrm{Furthermore,} \mathrm{CA}$ had a dose-dependent oxygen-reduction activity, which was higher compared with that of $\mathrm{VC}$ at the same concentration $(\mathrm{P}<0.05$; Fig. 1D).

Detection of the presence of peroxide provides initial evidence of rancidity in unsaturated dietary fat, with POV being the most widely used method. As displayed in Fig. 2, 0.02 and $0.05 \%$ CA significantly inhibited the oxidation of rapeseed oil on days 1,3 and 5 compared with that in the control group $(\mathrm{P}<0.05)$. Compared with the $0.02 \%$ TBHQ group, the anti-oxidation effect of 0.02 and $0.05 \% \mathrm{CA}$ was 
lower on days 1 and 3, while CA at $0.05 \%$ possessed a better inhibitory effect than that of TBHQ from the 5th day.

Anti-bacterial activity of $C A$. To test the influence of different concentrations of $\mathrm{CA}$ on the human intestinal microbiota, $\mathrm{CA}$ at 100,120 and $160 \mathrm{mM}$ was used to evaluate its effect on the growth of various beneficial microbes and pathogens of the microbiota. These concentrated were selected from previous studies and preliminary results $(10,13)$. As presented in Fig. 3, 100,120 and $160 \mathrm{mM}$ exerted no effect on the growth of the beneficial microbes L. plantarum, L. rhamnosus, L. crispatus, B. longum, while 120 and $160 \mathrm{mM} \mathrm{CA}$ significantly inhibited the growth of the pathogens $S$. enteritidis, S. typhimurium, E. coli and L. monocytogenes.

Anti-obesity activity of $C A$. Adipose tissue has an important role in maintaining lipid homeostasis and energy balance by storing triglycerides or liberating free fatty acids in response to changes in energy demands. As presented in Fig. 4, when CA was added prior to the induction of 3T3-L1, 12.5, 25 and $50 \mu \mathrm{M}$ CA significantly inhibited the formation of fat droplets, and the inhibitory effects of $50 \mu \mathrm{M}$ CA possessed a similar effect to that of metformin. However, when CA was added after induction, no obvious inhibition was observed in the 12.5 and $25 \mu \mathrm{M}$ CA groups, with only CA at $50 \mu \mathrm{M}$ achieving a significant inhibition of fat droplet formation in 3T3-L1 cells.

Anti-inflammatory activity of CA via suppressing pro-inflammatory cytokines. During inflammation, excess levels of cytokines (IL-1 $\beta$ and TNF- $\alpha$ ) damage cells and tissues, and may also activate macrophages and cause inflammation-associated diseases. To assess the anti-inflammatory effects of CA on the production of IL- $1 \beta$ and TNF- $\alpha$, RAW264.7 cells were treated with different concentrations of CA $(5,10,20$ or $40 \mu \mathrm{M})$. The results indicated that at all concentrations, CA significantly inhibited the LPS-induced production of IL-1 $\beta$ and TNF- $\alpha$ by RAW264.7 cells, and a dose-dependent effect of CA was observed (Fig. 5).

\section{Discussion}

Lipid oxidation is the oxidative degradation of lipids, which most frequently occurs in polyunsaturated fatty acids that contain multiple double bonds. Antioxidants, such as vitamin $\mathrm{C}$, are reducing agents acting as antioxidants and may reduce oxidizing substances, such as hydrogen peroxide; however, they also reduce metal ions that generate free radicals through the Fenton reaction (25). Plants and animals have developed complex and overlapping systems of oxidants and antioxidants to balance the oxidative state of components of their biomolecular system. The antioxidant activity of natural products is an area of current research.

Oxidative stress may be considered as either a cause or consequence of certain diseases; antioxidants are not only used as food additives to help protect against food deterioration, but also serve as antioxidant dietary supplements. Certain antioxidants, including $\beta$-carotene, vitamin $\mathrm{A}$, vitamin $\mathrm{E}$ and selenium, either individually or in combination, have been determined to be associated with survival $(25,26)$, and may lower the risk of cancer (27) and cardiovascular diseases (28).

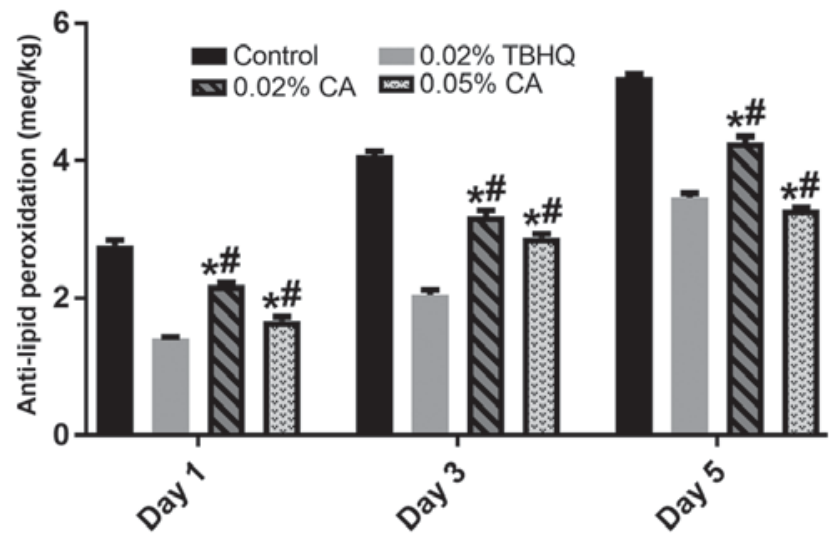

Figure 2. Anti-lipid peroxidation activity of different concentrations of CA. Values are expressed as the mean \pm standard deviation. ${ }^{*} \mathrm{P}<0.05$ vs. control group; ${ }^{\text {P }}<0.05$ vs. TBHQ group. $\mathrm{CA}$, chicoric acid; TBHQ, tert-butylhydroquinone; meq, milli-equivalents.

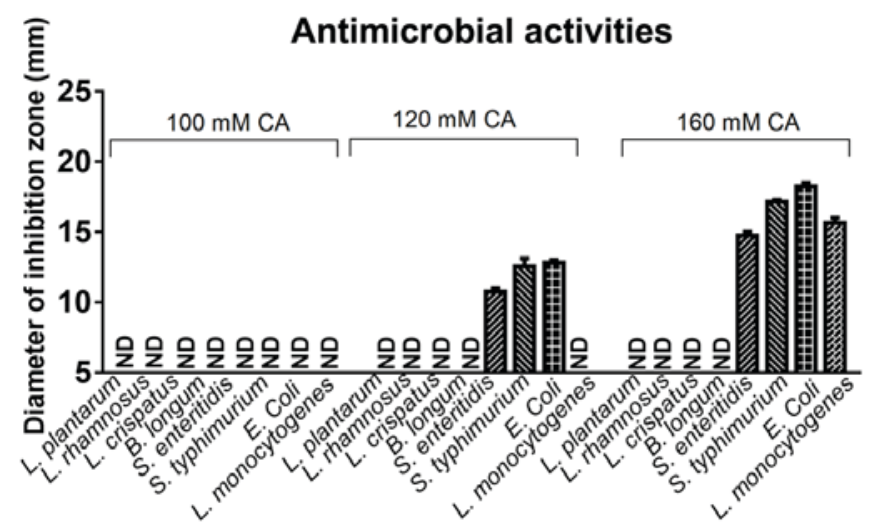

Figure 3. Anti-biotic activities of different concentrations of CA against selected bacterial strains. Values are expressed as the mean \pm standard deviation. ND, not detectable; CA, chicoric acid.

In the present study, the phytochemical CA, which possesses potent antioxidant and anti-inflammatory effects $(10,11)$, was assessed for its potential as a food antioxidant and to improve the health of humans.

First, the anti-oxidative activity of CA was evaluated. At all of the tested concentrations, CA exerted a marked anti-oxidative effect in terms of clearing $\cdot \mathrm{OH}, \mathrm{DPPH}$ and $\mathrm{Fe}^{2+}$, and displaying an oxygen-reducing activity, all of which was in a dose-dependent manner. Although according to most studies, $95 \mathrm{mg} / \mathrm{ml} \mathrm{VC}$ is sufficient for use as a positive control, 190 and $380 \mathrm{mg} / \mathrm{ml} \mathrm{VC}$ were also used in the present study to compare their anti-oxidative activity with that of the same doses of CA $(4,14)$. For $\cdot \mathrm{OH}$ and $\mathrm{Fe}^{2+}$ clearance, the results indicated that the anti-oxidative activity of $\mathrm{CA}$ was significantly lower than that of $\mathrm{VC}$ at the same concentrations $(\mathrm{P}<0.05)$, and that the clearance rate of $\cdot \mathrm{OH}$ and $\mathrm{Fe}^{2+}$ in the $380 \mathrm{mg} / \mathrm{ml}$ group was only 8.75 and $16.55 \%$, respectively, which was significantly lower than in the $95 \mathrm{mg} / \mathrm{ml}$ VC group $(\mathrm{P}<0.05)$. For DPPH, although the clearance of $\mathrm{CA}$ was significantly lower than that in the $\mathrm{VC}$ group at the same doses, $380 \mathrm{mg} / \mathrm{ml} \mathrm{CA}$ achieved a DPPH clearance of $47.8 \%$, which was significantly higher than that in the $95 \mathrm{mg} / \mathrm{ml} \mathrm{VC}$ group $(\mathrm{P}<0.05)$. Furthermore, the 95,190 and $380 \mathrm{mg} / \mathrm{ml} \mathrm{CA}$ 

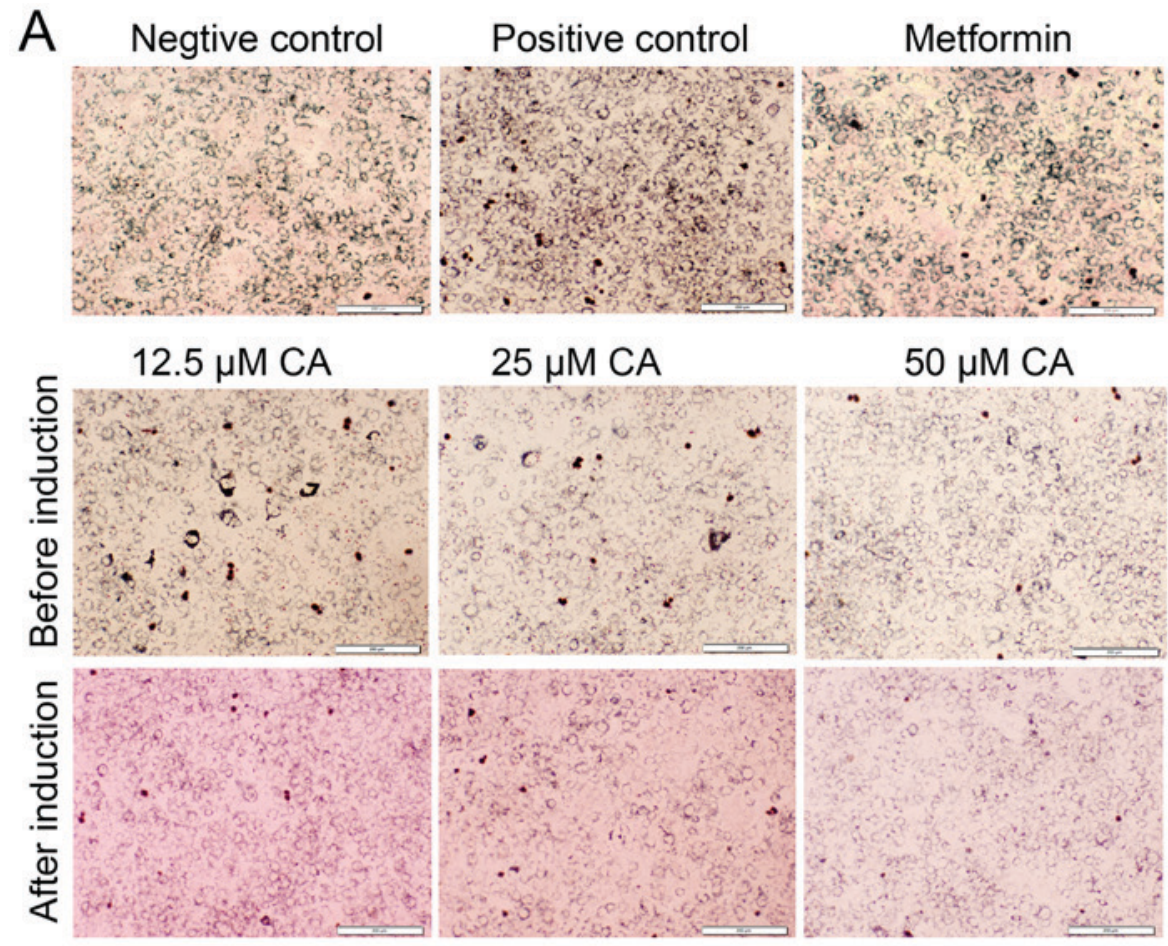

B

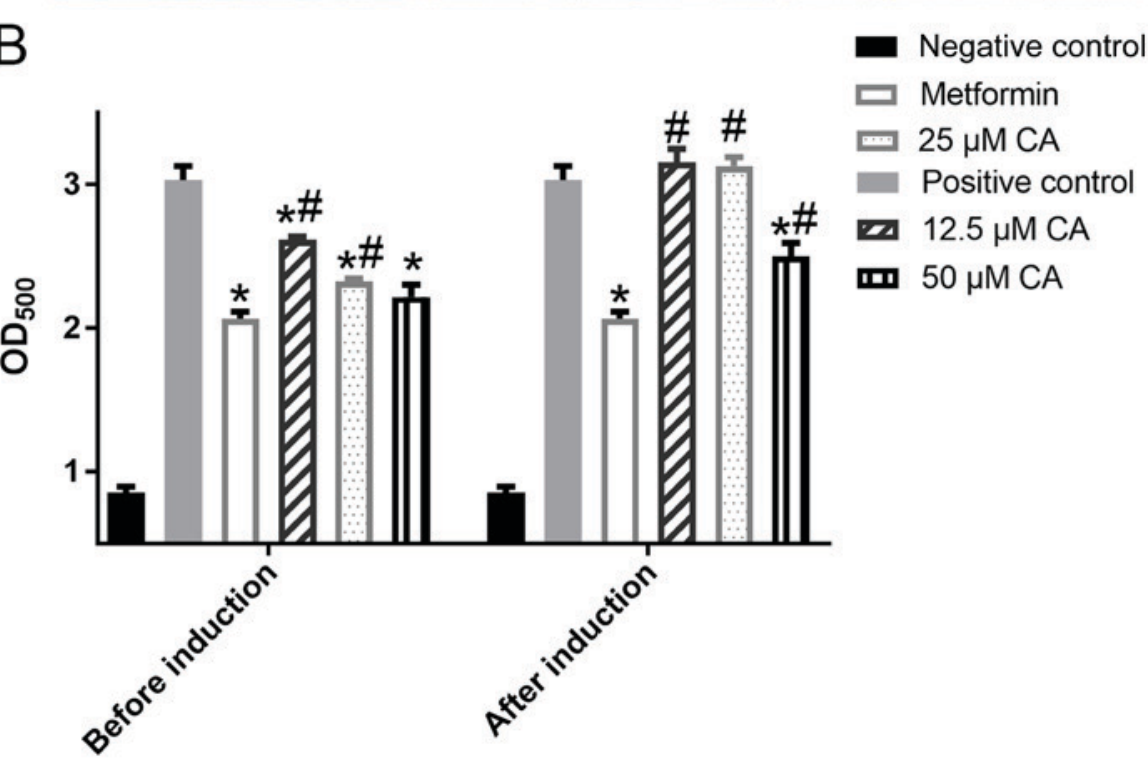

Figure 4. Inhibitory effects of the different concentrations of CA on lipid droplet formation in 3T3-L1 cells. (A) Oil red O staining results (magnification, x10; scale bar, $200 \mu \mathrm{m}$ ); (B) quantitative analysis of the Oil red O staining results. Values are expressed as the mean \pm standard deviation. "P $<0.05$ vs. positive control; ${ }^{"} \mathrm{P}<0.05$ vs. metformin group. $\mathrm{CA}$, chicoric acid; $\mathrm{OD}_{500}$, optical density at $500 \mathrm{~nm}$.

groups possessed a significantly higher oxygen-reduction activity than VC at the same dose, and $380 \mathrm{mg} / \mathrm{ml} \mathrm{CA}$ possessed a high oxygen-reduction activity of $1.87 \mu \mathrm{g} / \mathrm{ml}$ $(\mathrm{P}<0.05)$. Therefore, due to its high anti-oxidative activity, CA may have potential use a natural food antioxidant and may be used as an antioxidant to protect human health.

Autoxidation is a free radical reaction involving oxygen and leading to the deterioration of fats and oils, causing off-flavours and off-odours. The POV is useful to assess the extent to which spoilage has advanced. In the present study, 0.02 and $0.05 \%$ CA significantly reduced the POV compared with that in the control group at days 1,3 and $5(\mathrm{P}<0.05)$. Although the POV values for 0.02 and $0.05 \%$ CA at days 1 and 3 were significantly lower than those in the TBHQ group $(\mathrm{P}<0.05), 0.05 \% \mathrm{CA}$ achieved a significantly higher POV on day 5 than that in the $0.02 \%$ TBHQ group $(\mathrm{P}<0.05)$, indicating that the CA has a potent anti-oxidation effect on the oil and that it has a better long-term protection ability than TBHQ.

Numerous studies have indicated that intestinal microbes have an important role in human health $(20,29-32)$. Therefore, after confirmation of the antioxidant effect of $\mathrm{CA}$, the present study further investigated the potential effect of CA on common beneficial microbes and pathogens in the human microbiota. No anti-microbial activity of CA on L. plantarum, L. rhamnosus, L. crispatus and B. longum was observed at concentrations of 100,120 and $160 \mathrm{mM}$, while the concentration of $120 \mathrm{mM}$ obviously inhibited the growth of pathogens, 

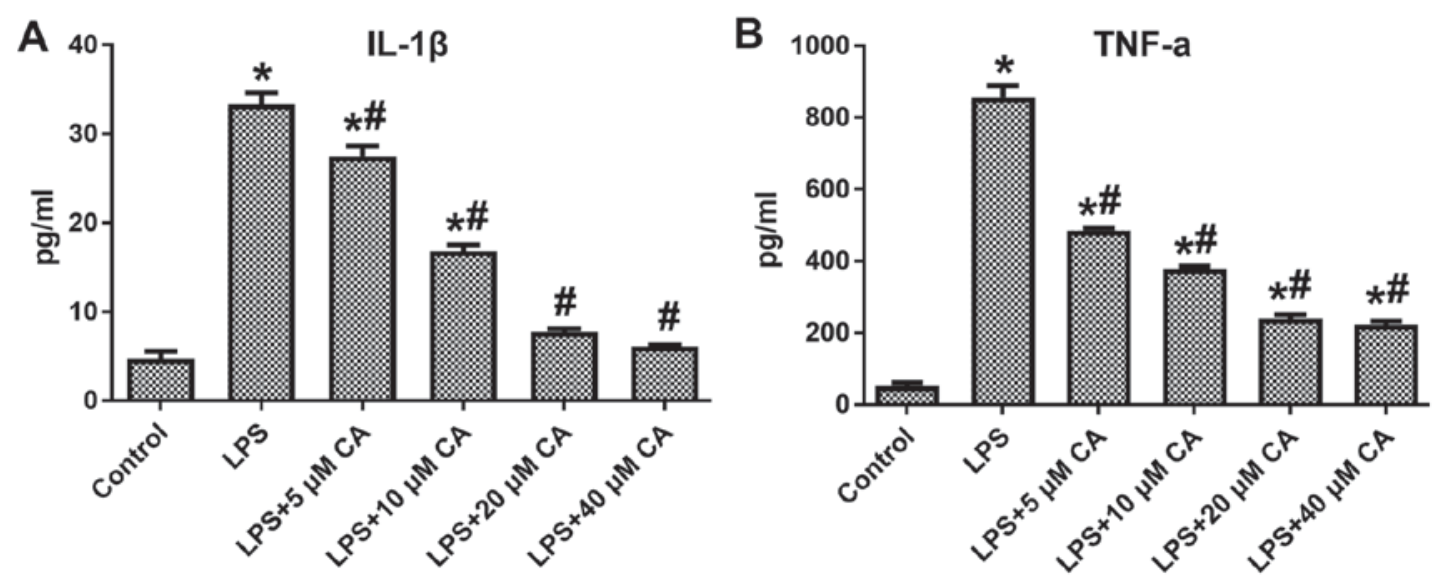

Figure 5. Effect of different concentrations of CA on the protein levels of (A) IL-1 $\beta$ and (B) TNF- $\alpha$ in LPS-induced RAW264.7 cells. Values are expressed as the mean \pm standard deviation. ${ }^{*} \mathrm{P}<0.05$ vs. control group; ${ }^{*} \mathrm{P}<0.05$ vs. LPS group. CA, chicoric acid; LPS, lipopolysaccharide; TNF, tumor necrosis factor; IL, interleukin.

including S.enteritidis, S. typhimurium and E. coli. In addition, $160 \mathrm{mM}$ CA had a potent inhibitory effect on all of the tested pathogens, namely $S$. enteritidis [inhibition zone diameter (IZD), $15 \mathrm{~mm}$ ], S. typhimurium (IZD, $17 \mathrm{~mm}$ ), E. coli (IZD, $18 \mathrm{~mm}$ ) and L. monocytogenes (IZD, $16 \mathrm{~mm}$ ). The absence of inhibitory effects of CA at a final concentration of $\sim 120 \mathrm{mM}$ on beneficial microbes of the healthy intestinal flora and its potent inhibitory effects on pathogens of the microbiota indicated that supplementation with CA may support human intestinal health.

The present study then assessed whether CA may be able to offset the harmful effects caused by excessive oil intake. For this, the effects of CA to inhibit intracellular lipid accumulation and inflammation were assessed using 3T3-L1 and RAW264.7 cells, respectively. In a preliminary experiment, the effects of different concentrations of CA on cell viability were assessed, revealing that $\mathrm{CA}$ at concentrations of $<50 \mu \mathrm{M}$ had no marked effect on the growth of 3T3-L1 cells and RAW264.7 cells (data not shown). When CA was added to the oil prior to adipogenic induction, 12.5, 25 and $50 \mu \mathrm{M}$ CA significantly inhibited fat droplet formation compared with that in the positive control group $(\mathrm{P}<0.05)$, with $50 \mu \mathrm{M}$ CA having a similar effect to that of metformin. However, when CA was added to the oil after induction, only $50 \mu \mathrm{M}$ CA significantly inhibited the fat droplet formation compared with that in the positive control group $(\mathrm{P}<0.05)$, and its inhibitory effects were significantly lower than those in the metformin group. Oil red $\mathrm{O}$ staining indicated that although a high dose of CA obviously reduced the presence of fat droplets even after they had been formed, and was therefore able to break down the fat droplets, only low doses achieved a significant inhibition if CA was added prior to adipogenic induction to inhibit droplet formation. The desired effect of CA to inhibit the formation of fat droplets also confirmed that CA treatment may reduce the likelihood of obesity in healthy individuals. Therefore, when CA is used as a natural food antioxidant and mixed with oil to enter the human intestine, it may potentially be able to effectively inhibit the harmful effects of excessive oil intake.

As is already known, excess levels of IL-1 $\beta$ and TNF- $\alpha$ produced by activated eosinophils, macrophages, mononuclear phagocytes and neutrophils cause damage to cells and tissues, which eventually causes inflammation-associated diseases; macrophages have an important role in regulating several immunopathological conditions and inducing the overexpression of pro-inflammatory mediators of the inflammatory process (15). Therefore, RAW264.7 cells were used in the present study. In the present study, CA at 5, 10, 20 and $40 \mu \mathrm{M}$ significantly reduced the production of IL-1 $\beta$ and TNF- $\alpha$ compared with that in the LPS group $(\mathrm{P}<0.05)$, with their effect being dose-dependent. In addition, $20 \mu \mathrm{M}$ CA was sufficient to restore IL-1 $\beta$ to near-normal levels. As CA exerted potent anti-inflammatory effects by inhibiting pro-inflammatory cytokines, it is indicated that it may efficiently prevent diseases caused by chronic inflammation.

To date, only few studies have been performed to systematically evaluate the role of CA as an antioxidant oil additive, as well as its potential benefits on human health involving immunity, obesity and the intestinal microbiota. The potent anti-oxidative activity (prolongation of the shelf life of oil), anti-bacterial activity (inhibition of the proliferation of pathogenic microbes in the host's intestines), anti-inflammatory effects and inhibition of intracellular triglyceride accumulation by CA suggest that it may promote intestinal and overall health. CA has potential use as a functional natural food antioxidant to prevent the oxidation of oil, protect human intestinal health, delay senescence and prevent chronic diseases.

\section{Acknowledgements}

Not applicable.

\section{Funding}

The present study was supported by grants from the Open Foundation of Hubei Key Laboratory of Lipid Chemistry and Nutrition (grant no. 201602), the National Natural Science Foundation of China (grant nos. 31560264, 81503364), and grants from Jiangxi Province (grant nos. 20171BCB23028 and 20175526). 


\section{Availability of data and materials}

All data generated or analyzed during this study are included in this published article.

\section{Authors' contributions}

TC and MF designed the present study; XZ, FH and XX performed the experiments; MF and TC analyzed all data and wrote the manuscript. All authors read and approved the final manuscript.

\section{Ethics approval and consent to participate}

The present study was approved by the Ethics Committee of the Second Affiliated Hospital of Nanchang University (Nanchang, China), and the sampling of human specimens was performed after obtaining consent from the patients or volunteers.

\section{Patient consent for publication}

Patients or their guardians provided written informed consents for publication.

\section{Competing interests}

The authors declare that they have no competing interests.

\section{References}

1. Willett WC: Is dietary fat a major determinant of body fat? Am J Clin Nutr 67 (Suppl 3): 556S-562S, 1998.

2. Balabanova B and Gulaboski R: Human health risks from heavy metals via consumption of contaminated food. In: International Symposium at Faculty of Medical Sciences. http://eprints.ugd. edu.mk/14904/ Accessed November 24, 2015.

3. Morita M, Naito Y, Yoshikawa T and Niki E: Antioxidant capacity of blueberry extracts: Peroxyl radical scavenging and inhibition of plasma lipid oxidation induced by multiple oxidants. J Berry Res 7: 1-9, 2017.

4. Niki E: Oxidative stress and antioxidants: Distress or eustress? Arch Biochem Biophys 595: 19-24, 2016.

5. Niki E: Biomarkers of lipid peroxidation in clinical material. Biochim Biophys Acta 1840: 809-817, 2014.

6. Prior RL, Sintara M and Chang T: Multi-radical (ORACMR5) antioxidant capacity of selected berries and effects of food processing. J Berry Res 6: 159-173, 2016.

7. Ivanova-Petropulos V, Hermosín-Gutiérrez I, Boros B, et al: Phenolic compounds and antioxidant activity of macedonian red wines. J Food Compos Anal 41: 1-14, 2015.

8. Forbeshernandez TY, Gasparrini M, Afrin S, Bompadre S, Mezzetti B, Quiles JL, Giampieri F and Battino M: The healthy effects of strawberry polyphenols: Which strategy behind antioxidant capacity? Crit Rev Food Sci Nutr 1 (Suppl 56): S46-S59, 2016

9. Kader AA, Zagory D and Kerbel EL: Modified atmosphere packaging of fruits and vegetables. Crit Rev Food Sci 28: 1-30, 1989.

10. Xiao H, Wang J, Yuan L, Xiao C, Wang Y and Liu X: Chicoric acid induces apoptosis in 3T3-L1 preadipocytes through ROS-mediated PI3K/Akt and MAPK signaling pathways. J Agric Food Chem 61: 1509-1520, 2013.

11. Liu Q, Chen Y, Shen C, Xiao Y, Wang Y,Liu Z and Liu X: Chicoric acid supplementation prevents systemic inflammation-induced memory impairment and amyloidogenesis via inhibition of NF-кB. FASEB J 31: 1494-1507, 2017.
12. Park CM, Jin KS, Lee YW and Song YS: Luteolin and chicoric acid synergistically inhibited inflammatory responses via inactivation of PI3K-Akt pathway and impairment of NF- $\kappa \mathrm{B}$ translocation in LPS stimulated RAW 264.7 cells. Eur J Pharmacol 660: 454-459, 2011.

13. Xiao H, Xie G, Wang J, et al: Chicoric acid prevents obesity by attenuating hepatic steatosis, inflammation and oxidative stress in high-fat diet-fed mice. Food Res Int 54: 345-353, 2013.

14. Lai LS, Chou ST, and Chao WW: Studies on the antioxidative activities of hsian-tsao (mesona procumbens hemsl) leaf gum. J Agric Food Chem 49: 963-968, 2001.

15. Jiang M, Deng K, Jiang C, Fu M, Guo C, Wang X, Wang X, Meng F, Yang S, Deng K, et al: Evaluation of the antioxidative, antibacterial, and anti-inflammatory effects of the aloe fermentation supernatant containing lactobacillus plantarum HM218749.1. Mediators Inflamm 2016: 2945650, 2016.

16. Dorman HJD, Peltoketo A, Hiltunen R and Tikkanen MJ: Characterisation of the antioxidant properties of de-odourised aqueous extracts from selected lamiaceae herbs. Food Chem 83: 255-262, 2003.

17. Cheng-Lun L, Derong T and Le L: Research on the extracting and anti-oxidation dynamic characteristics of ginger oleoresin. Int J Food Sci Tech 43: 517-525, 2008

18. Chen T, Wu Q, Li S, Xiong S, Jiang S, Tan Q, Zhang Z, Zhu D and Wei H: Microbiological quality and characteristics of probiotic products in china. J Sci Food Agric 94: 131-138, 2014

19. Meng F, Chen T, Wang X, Wang X, Wei H, Tian P, Wang H, Zhao X, Shen L and Xin H: Evaluation of the accuracy and sensitivity of high-throughput sequencing technology using known microbiota. Mol Med Rep 17: 408-413, 2018.

20. Chen T, Wu Q, Zhou H, et al: Assessment of commercial probiotic products in china for labelling accuracy and probiotic characterisation of selected isolates. Int J Dairy Technol 70: 119-126, 2017.

21. Wang X, Wu Q, Deng K, Wei Q, Hu P, He J, Liu H, Zheng Y, Wei H, Shah NP and Chen T: A novel method for screening of potential probiotics for high adhesion capability. J Dairy Sci 98: 4310-4317, 2015.

22. Deng K, Chen T, Wu Q, Xin H, Wei Q, Hu P, Wang X, Wang X, Wei $H$ and Shah NP: In vitro and in vivo examination of anticolonization of pathogens by lactobacillus paracasei $\mathrm{FJ} 861111.1$. J Dairy Sci 98: 6759-6766, 2015.

23. Lim SM, Goh YM, Kuan WB and Loh SP: Effect of germinated brown rice extracts on pancreatic lipase, adipogenesis and lipolysis in 3T3-L1 adipocytes. Lipids Health Dis 13: 169, 2014.

24. Wójcik M, Wessely-Szponder J, Cichoż-Lach H, Celiński K and Bobowiec R: In vitro proinflammatory polarization of macrophages isolated from hepatocarcinogenic stage in humans and rats. In Vivo 30: 853-862, 2016.

25. Carr A and Frei B: Does vitamin $\mathrm{C}$ act as a pro-oxidant under physiological conditions? FASEB J 13: 1007-1024, 1999.

26. Abner EL, Schmitt FA, Mendiondo MS, Marcum JL and Kryscio RJ: Vitamin E and all-cause mortality: A meta-analysis. Curr Aging Sci 4: 158-170, 2011.

27. Caraballoso M, Sacristan M, Serra C and Bonfill X: Drugs for preventing lung cancer in healthy people. Cochrane Database Syst Rev 10: CD002141, 2003.

28. Rees K, Hartley L, Day C, Clarke A and Stranges S: Selenium supplementation for the primary prevention of cardiovascular disease. Cochrane Database Syst Rev 1012: CD009671, 2012.

29. Zhao X, Chen T, Meng F, Wang H, Tian P, Tang X, Wang X, Wang $\mathrm{X}$, Xin $\mathrm{H}$ and Wei $\mathrm{H}$ : Therapeutic effect of herb residue fermentation supernatant on spleenâ-'deficient mice. Mol Med Rep 17: 2764-2770, 2018

30. Zhang F, Zhang M, Wang Y, Li C and Chen T: Comparison of the common bacteria in human and mouse tumours using high-throughput sequencing. Mol Med Rep 17: 6717-6722, 2018.

31. Chen T, Yan S, Wang X, Wang X, Meng F, Yang S, Yang J and Xin $\mathrm{H}$ : High-throughput sequencing analyses of oral microbial diversity in healthy people and patients with dental caries and periodontal disease. Mol Med Rep 16: 127-132, 2017.

32. Chen H, Luo T, Chen T and Wang G: Seminal bacterial composition in patients with obstructive and non-obstructive azoospermia. Exp Ther Med 15: 2884-2890, 2018. 